\title{
Volume residual gástrico como verificação de tolerância alimentar enteral em recém-nascidos de baixo peso e lactentes: revisão da literatura
}

\author{
Gastric residual volume as enteral feeding evaluation in low \\ birth weight infants and infants: literature review
}

Luís Cláudio Santos Pinto', Mauro de Souza Pantoja', Marcus Vinícius Henriques Brito', Mariela Carolina Santos Carballo'

Resumo Introdução: A ocorrência de intolerância à iniciação dietética lipídica e proteica enteral em prematuros é comum. Esta dieta enteral deve ser iniciada, ao mesmo tempo, de forma gradual e precoce para evitar a depleção nutricional, a qual poderá levar a uma condição de desnutrição se não for tratada. Para determinar se a nutrição enteral está progredindo de forma satisfatória, existem certos parâmetros como o volume residual gástrico (VRG), usado para determinar se recém-nascidos de baixo peso (RNBP), de muito baixo peso (RNMBP) e lactentes se mostram tolerantes à progressão deste modelo dietético. Objetivo: realizar uma revisão da literatura médica, em bases de dados indexadas, para verificar se a determinação do VRG se mostra como ferramenta eficaz na predição da correta progressão da dieta enteral em RNBP, RNMBP e lactentes. Método: Verificar a importância da utilização do VRG como ferramenta de acompanhamento nutricional os autores buscaram artigos com as palavras-chave: esvaziamento gástrico, recém-nascido, baixo peso, lactente. Foram encontrados 47 artigos em três bases de dados científicas em um período de busca entre fevereiro e abril de 2018: Pubmed, Portal da Biblioteca Virtual em Saúde (LILACS e Medline) e Biblioteca Cochrane. Apenas vinte e três trabalhos foram considerados por apresentarem a VRG como método para verificar a tolerância dietética enteral em RNBP, RNMBP e lactentes. Nenhum artigo foi excluído pelos parâmetros de idioma ou data de publicação. Conclusão: Apesar de ser uma prática rotineira nos serviços de unidades de terapia intensiva neonatal verificar a tolerância à alimentação enteral no RNBP e lactentes pelo VRG, não há consenso entre os diferentes protocolos de serviço encontrados na literatura, não sendo possível, no momento, estabelecer o VRG como um parâmetro validado na avaliação da progressão dietética enteral nessa população.

Descritores: esvaziamento gástrico; recém-nascido de baixo peso; lactente.

Summary Introduction: The occurrence of lipid and protein enteral dietary initiation intolerance in preterm is common. This enteral dietary should be initiated, at the same time, in a gradually and earliest way to avoid depletion, which one will lead a malnutrition condition if untreated. To determine if enteral nutrition is being successfully performed, there are certain parameters like residual gastric volume (RGV) that are used to determine if low weight newborns (LWN) and infants are tolerant to enteral dietary progression. Objective: Searching in actual literature data that can confirm if RGV is a reliable tool to validate enteral dietary tolerance progress. Methods: To evaluate the importance of using RVG as a nutritional monitoring tool the authors searched papers with the keywords: gastric emptying, newborn, low-weight, infant. Forty-seven papers were found in three scientific data base in a search period between February-April 2018: Pubmed, Portal da Biblioteca Virtual em Saúde (Medline and LILACS) and Cochrane Library, although, only twenty three papers were considered in this review by presenting RGV as a method to verify enteral dietary tolerance in LWN and infants. No papers were excluded by language or publishing date. Conclusion: Despite is a routine practice in neonatal intensive care units services to verify enteral feeding tolerance in LWN and infants by RGV, there is no consensus among these different services protocols and is not possible, at the moment, establishes RGV as a reliable parameter for enteral dietary progression evaluation in this population.

Keywords: gastric emptying; low birth weight newborn; infant.

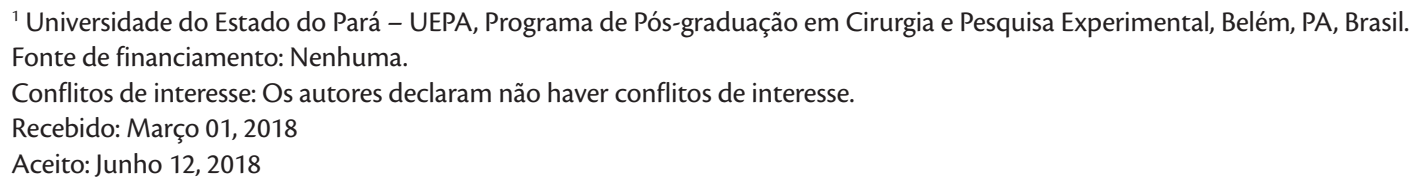

Trabalho realizado no Laboratório de Cirurgia Experimental, Universidade do Estado do Pará - UEPA, Belém, PA, Brasil. 


\section{Introdução}

Ainda hoje as taxas de ocorrência de baixo peso ao nascer (BPN) são bastante oscilantes entre países desenvolvidos e em desenvolvimento, cada um com suas peculiaridades ${ }^{1}$.

Nos países desenvolvidos, as taxas de BPN se dão principalmente pela ocorrência de partos prematuros, enquanto que nos países em desenvolvimento são em sua maior parte consequência do retardo de crescimento intrauterino (RCIU) decorrente de condições socioeconômicas desfavoráveis ${ }^{1}$.

Devido à imaturidade do trato gastrointestinal do prematuro, grande é a ocorrência de intolerância ao início da dieta enteral, que deve ser instituída paulatinamente, porém o mais precoce possível para que se evite depleção lipídica e proteica que, se não tratadas, levarão a um estado de desnutrição².

Para que se verifique que a nutrição enteral esteja sendo realizada com sucesso, existem determinados parâmetros utilizados para determinar se o recém-nascido de baixo peso (RNBP) está tolerante á progressão da dieta enteral, entre eles estão o volume residual gástrico aferido por sonda ou ultrassom e a medida da circunferência abdominal ${ }^{3-8}$.

Como exemplo, Murase et al. ${ }^{9}$ avaliaram em seu estudo que RNBP com leucomalácia ventricular cística se mostraram com maior intolerância alimentar enteral por apresentarem maior distensão abdominal e maior volume residual gástrico quando comparados ao grupo controle. Também Ozer et al. ${ }^{10}$ estudaram o papel do GLP-2 (glucagon like peptide-2), um peptídeo gastrointestinal com ações endócrinas e parácrinas com papel trófico no esvaziamento gástrico, de RNBP e utilizaram como método para avaliação de intolerância alimentar os valores de distensão abdominal e o volume residual gástrico (VRG).

Poucos são os artigos encontrados na literatura que sugerem uma real eficácia de predição destes parâmetros para que se determine uma resposta adequada do RNBP e lactentes á progressão da dieta enteral, sendo o objetivo deste trabalho reunir estes artigos e verificar se há uma possível tendência de concordância entre eles.

\section{Método}

Para verificar a importância da utilização do VRG como ferramenta de acompanhamento nutricional, realizou-se esta revisão de literatura onde foram buscados artigos nas bases de dados Pubmed, Portal Regional da Biblioteca Virtual em Saúde (Medline e LILACS) e Cochrane Library, no período de fevereiro a abril de 2018 e que apresentavam como descritores: nutrição enteral, esvaziamento gástrico, volume residual, recém-nascido, recém-nascido de baixo peso, recém-nascidos de muito baixo peso e lactente.

Devido ao fato de que foram achados poucos artigos na literatura que abordam este tema, não houve exclusão de artigos por data de publicação e nem por idioma, sendo que todos os artigos encontrados foram redigidos na língua inglesa ou em português. Na presente busca, não houve artigos escritos em espanhol.

Um total de 47 artigos foram encontrados em ocasião da pesquisa (29 no Pubmed, 18 no Portal da Biblioteca Virtual em Saúde, onde 7 eram referências repetidas encontradas no Pubmed e nenhum artigo na base de dados da Cochrane Library foi encontrado), dos quais apenas 23 foram utilizados por apresentarem, de acordo com avaliação crítica dos autores, concordância direta na utilização do VRG (esvaziamento gástrico) como método único ou associado a outros parâmetros na avaliação da adequação e progressão da alimentação enteral em RNBP, RNMBP e lactentes.

Os 24 artigos excluídos, apesar de apresentarem os descritores em seu conteúdo, não utilizavam o uso do VRG como método para avaliar alimentação enteral dentro dos objetivos e população específicos adotados pelos autores para esta revisão.

\section{Resultados e discussão}

De acordo com a literatura encontrada, a verificação do volume residual gástrico vem sendo utilizada como rotina para determinação de tolerância à nutrição enteral em RNBP. Porém, apesar de rotineira, esta prática ainda apresenta muita contradição entre os autores.

Kaur et al. ${ }^{3}$ compararam a circunferência abdominal (CA) pré-alimentação e o volume residual gástrico (VRG) como medidas de intolerância alimentar enteral em RNMBP. Verificaram que o grupo que utilizou a medida da circunferência abdominal conseguiu atingir a alimentação plena $(180 \mathrm{ml} / \mathrm{Kg} /$ dia $)$ em menor número de dias quando comparado ao grupo de medida do VRG (média de 10 versus 14 dias respectivamente). Além disso, o grupo VRG apresentou maior número de interrupções na progressão da 
dieta enteral do que o grupo CA, inferindo então que a medida da CA é melhor parâmetro para avaliar tolerância alimentar que o VRG.

Também Malhotra et al. ${ }^{11}$ verificaram em seu estudo que o aumento do valor da circunferência abdominal a partir de $2 \mathrm{~cm}$ deve ser levado em consideração como um fator preditivo para a redução ou até mesmo suspensão da alimentação enteral em recém-nascidos pré-termo.

Li et al. ${ }^{4}$ afirmam que a medida do VRG é prática comum nos serviços de UTI neonatal (UTI N) para verificar tolerância alimentar dos RNMBP, entretanto esta prática não apresenta homogeneidade entre diferentes serviços, o que dificulta estabelecer um protocolo que realmente possa confirmar o valor do VRG para a tomada de decisão em evoluir com a dieta enteral.

Existem diferenças entre autores quanto a que valor de VRG aceitar como bom preditor de tolerância à alimentação. Moody et al. ${ }^{12}$ ao avaliarem a tolerância alimentar enteral à adição de fortificante ao leite materno para RNMBP, definiram que um VRG $>2 \mathrm{ml} / \mathrm{Kg}$ ou volume acima de $50 \%$ do total da alimentação oferecida com 3 horas prévias à medição seria um bom parâmetro para determinar intolerância à progressão da dieta enteral, enquanto Mihatsh et al. ${ }^{13}$ definiram o valor de VRG $>3 \mathrm{ml}$ como intolerância à alimentação enteral em RNMBP pesando até 750 gr. Já Kairamkonda et al. ${ }^{14}$ utilizaram VRG $>50 \%$ do volume total da alimentação oferecida com 4 horas prévias à avaliação, em duas ocasiões consecutivas.

Utiliza-se também o VRG como método de avaliação quanto ao regime da alimentação enteral a ser escolhido em RNBP, se por infusão gástrica contínua ou por bolus intermitente. Dollberg et al. ${ }^{15}$ afirmaram que a infusão por bolus intermitente foi superior, porém Horn et al. ${ }^{16}$ não foram capazes de demonstrar superioridade de um método em relação ao outro.

Gonzalez et al. ${ }^{17}$ estudaram qual seria a melhor temperatura para se oferecer leite aos RNBP e concluíram que o grupo alimentado por gavagem com leite em temperatura morna apresentou menores valores de VRG quando comparado aos grupos leite quente e leite frio.

Já Silva e Collares ${ }^{18}$ estudaram qual seria a melhor posição para manter o recém-nascido e o lactente durante alimentação enteral. Para isso utilizaram sonda nasogástrica com uma solução de hidratação oral e mediram o VRG após a alimentação nas posições livre (sem contenção), supina, decúbito direito e decúbito esquerdo. Concluiram que o decúbito lateral esquerdo esteve relacionado com maior VRG significando pior esvaziamento gástrico.

Também se utilizou do valor do VRG em trabalho de Tekgündüz et al. ${ }^{19}$ para avaliar se havia melhora da tolerância da alimentação enteral em RNBP com massagens abdominais de 15 minutos de duração, duas vezes ao dia antes da alimentação, durante 5 dias. Houve menor VRG com diferença estatística significante do grupo massagem quando comparado ao grupo controle.

A necessidade em se avaliar o risco de aspiração de conteúdo gástrico devido anestesia geral em lactentes se constitui como importante questão. Desta forma, Litman et al. ${ }^{20}$ determinaram que oferecer líquidos claros aos lactentes no período pré-operatório, quando comparado ao leite materno, melhorou a tolerância alimentar diminuindo o VRG, sugerindo menor risco de aspiração com líquidos claros.

Wakayama, Wilkins e Kimura ${ }^{21}$ estudaram qual seria o tipo de alimentação enteral mais adequada para evitar aspiração em lactentes no pós-operatório imediato de herniorrafia inguinal. Compararam bebida láctea a 15\% com solução de dextrose a 5\%, sendo os parâmetros utilizados: ph e osmolaridade do conteúdo gástrico, clearance gástrico e VRG em 15, 30 e 60 minutos após alimentação. Neste estudo não houve desvantagens significativas em se oferecer a bebida láctea quando comparada à solução de dextrose a $15 \%$.

A frequência de verificações de VRG, sua coloração e a decisão de quando descartar ou retomar suas medições não contam com um padrão a ser seguido, fato também observado em estudo de Mihatsh et al. ${ }^{13}$, Martinez et al..$^{22}$ e Torrazza et al..$^{23}$

Observa-se, portanto, que a aferição do VRG está presente na metodologia de vários estudos sobre intolerância alimentar enteral e esvaziamento gástrico em RNBP, RNMBP e lactentes, porém não existe uniformidade na forma de como este parâmetro é avaliado para que se possa garantir um protocolo que defina intolerância alimentar a partir de dados sobre o VRG de forma conclusiva. Desta forma, se faz necessário que novos estudos possam ser realizados para que se estabeleça uma comparação metodológica entre eles e assim comprovar de que forma o VRG pode ser utilizado de forma mais confiável para avaliar intolerância alimentar. 


\section{Conclusão}

A partir da literatura estudada, não foi possível determinar concordância entre os diferentes métodos utilizados para avaliar VRG e tolerância alimentar enteral em RNBP e lactentes.

\section{Referências}

1. Pedraza DF. Baixo peso ao nascer no Brasil: revisão sistemática de estudos baseados no sistema de informações sobre nascidos vivos. Revista de Atenção à Saúde. 2014;41(12):37-50.

2. Briassoulis G, Zavras N, Hatzis T. Malnutrition, nutritional indices, and early enteral feeding in critically ill children. Nutrition. 2001;17(78):548-57. http://dx.doi.org/10.1016/S0899-9007(01)00578-0. PMid:11448572.

3. Kaur A, Kler N, Saluja S, Modi M, Soni A, Thakur A, et al. Abdominal circumference or gastric residual volume as measure of feed intolerance in VLBW infants. J Pediatr Gastroenterol Nutr. 2015;60(2):259-63. http://dx.doi.org/10.1097/MPG.0000000000000576. PMid:25238118.

4. Li Y-F, Lin H-C, Torrazza RM, Parker L, Talaga E, Neu J. Gastric residual evaluation in preterm neonates: a useful monitoring technique or a hindrance? Pediatr Neonatol. 2014;55(5):335-40. http://dx.doi.org/10.1016/j.pedneo.2014.02.008. PMid:25129325.

5. Perrella SL, Hepworth AR, Simmer KN, Geddes DT. Validation of ultrasound methods to monitor gastric volume changes in preterm infants. J Pediatr Gastroenterol Nutr. 2013;57(6):741-9. http://dx.doi.org/10.1097/MPG.0b013e3182a938d7. PMid:23969536.

6. Shulman RJ, Ou C-N, Smith EO. Evaluation of potential factors predicting attainment of full gavage feedings in preterm infants. Neonatology. 2011;99(1):38-44. http://dx.doi.org/10.1159/000302020. PMid:20588069.

7. Liang Z, Lu L, Zhou Y, Chen S, Huang Y, Lin Z. Clinical investigation of formula feeding in extremely low birth weight infants. Zhonghua Er Ke Za Zhi. 2014;52(1):51-6. PMid:24680409.

8. Heimann K, Schoberer M, Posielek J, Fitzner C, Orlikowsky T. The ratio of abdominal circumference and body weight-an objective parameter for the daily ward round in a NICU? Klin Padiatr. 2014;226(2):72-5. http://dx.doi.org/10.1055/s-0034-1368718. PMid:24633977.

9. Murase M, Ishida A, Momota T. Early gastrointestinal function and nutritional status in preterm infants with cystic periventricular leukomalacia. Biol Neonate. 2002;82(2):78-83. http://dx.doi.org/10.1159/000063091. PMid:12169828.

10. Ozer EA, Holst JJ, Duman N, Kumral A, Ozkan H. The relationship between glucagon-like peptide 2 and feeding intolerance in preterm infants. J Trop Pediatr. 2009;55(4):276-7. http://dx.doi.org/10.1093/tropej/fmn033. PMid:18499738.

11. Malhotra AK, Deorari AK, Paul VK, Bagga A, Meharban S. Gastric residuals in preterm babies. J Trop Pediatr. 1992;38(5):262-4. http://dx.doi. org/10.1093/tropej/38.5.262. PMid:1433454.

12. Moody GJ, Schanler RJ, Lau C, Shulman RJ. Feeding tolerance in premature infants fed fortified human milk. J Pediatr Gastroenterol Nutr. 2000;30(4):408-12. http://dx.doi.org/10.1097/00005176-200004000-00011. PMid:10776952.

13. Mihatsch WA, von Schoenaich P, Fahnenstich $H$, Dehne N, Ebbecke $H$, Plath $C$, et al. The significance of gastric residuals in the early enteral feeding advancement of extremely low birth weight infants. Pediatrics. 2002;109(3):457-9. http://dx.doi.org/10.1542/peds.109.3.457. PMid:11875141.

14. Kairamkonda VR, Deorukhkar A, Bruce C, Cooms R, Fraser R, Mayer APT. Amylin peptide is increased in preterm neonates with feed intolerance. Child Fetal Neonatal Ed. 2008; 93(4):65-70.

15. Dollberg S, Kuint J, Mazkereth R, Mimouni FB. Feeding tolerance in preterm infants: randomized trials of bolus and continuous feeding. J Am Coll Nutr. 2000;19(6):797-800. http://dx.doi.org/10.1080/07315724.2000.10718080. PMid:11194534.

16. Horn D, Chaboyer W, Schluter PJ. Gastric residual volume in critically ill paediatric patients: a comparison of feeding regimens. Aust Crit Care. 2004;17(3):98-100, 102-3. http://dx.doi.org/10.1016/S1036-7314(04)80011-0. PMid:15493856.

17. Gonzales I, Duryea EJ, Vasquez E, Geraghty N. Effect of enteral feeding temperature on feeding tolerance in peterm infants. Neonatal Netw. 1995;14(3):39-43. PMid:7603419.

18. Silva PE, Collares EF. Esvaziamento gástrico em crianças: Influência da posição do corpo, utilizando como refeição de prova uma solução hidratante de uso oral. Arq Gastroenterol. 1988;25(2):104-9. PMid:3255271.

19. Tekgündüz KŞ, Gürol A, Apay SE, Caner I. Effect of abdome massage for prevention of feeding intolerance in preterm infants. Ital J Pediatr. 2014;40(1):89. http://dx.doi.org/10.1186/s13052-014-0089-z. PMid:25394549.

20. Litman RS, Wu CL, Quinlivan JK. Gastric volume and ph in infants fed clear liquids and breast milk prior to surgery. Anesth Analg. 1994;79(3):482-5. http://dx.doi.org/10.1213/00000539-199409000-00013. PMid:8067551.

21. Wakayama Y, Wilkins S, Kimura K. Is $5 \%$ dextrose in water a proper choice for initial postoperative feeding in infants? J Pediatr Surg. 1988 jul;23(7):644-6. http://dx.doi.org/10.1016/S0022-3468(88)80637-7. PMid:3204463. 
22. Martinez EE, Bechard LJ, Mehta NM. Nutrition algorithms and bedside nutriente delivery practices in pediatric intensive care units: an international cohort study. Nutr Clin Pract. 2014;29(3):360-7. PMid:24740498.

23. Torrazza RM, Parker LA, Li Y, Talaga E, Shuster J, Neu J. Aspiration and evaluation of gastric residuals in the neonatal intensive care unit: state of the science. J Perinatol. 2015;29(1):5159.

\section{Autor correspondente}

Luís Cláudio Santos Pinto

Universidade do Estado do Pará - UEPA, Programa de Pós-graduação em Cirurgia e Pesquisa Experimental

Travessa Pirajá, Conjunto Santos Dumont II, casa 23, Marco

CEP 66087-490, Belém, PA, Brasil

Tel.: (91) 98114-8982

E-mail: Icopinto@uol.com.br

Informação sobre os autores:

LCSP e MCSC são mestrandos do Programa de Mestrado em Cirurgia e Pesquisa Experimental - CIPE, Universidade do Estado do Pará - UEPA. MSP é professor do Programa de Mestrado em Cirurgia e Pesquisa Experimental - CIPE, Universidade do Estado do Pará - UEPA.

MVHB é coordenador do Programa de Mestrado em Cirurgia e Pesquisa Experimental - CIPE, Universidade do Estado do Pará - UEPA.

\section{Contribuição dos autores}

MCSC e LCSP foram redatores do artigo, alunos do Mestrado CIPE; MSP foi orientador proponente do tema, professor do programa do Mestrado CIPE; MVHB foi orientador coordenador do programa do mestrado CIPE. 